\title{
Contact Loss Simulator to Analyze the Contact Loss of a Rigid Catenary System
}

\author{
No-Geon Jung* and Jae-Moon Kim ${ }^{\dagger}$
}

\begin{abstract}
In this paper, a contact loss simulator for a rigid catenary system was designed and used to analyze the effect on the power source according to the conditions of the rigid catenary system and pantograph. R-bar applied to a high-speed train among the real rigid catenary system was used in the contact loss simulator for rigid catenary systems. The excitation frequency generated with the movement of the railway vehicle was simulated. The characteristics according to the frequency and amplitude of the excitation frequency and the presence or absence of pantograph movement were analyzed. This work is considered to be helpful in analyzing the characteristics of contact loss in the interface between a real rigid catenary system and a rail vehicle.
\end{abstract}

Keywords: Contact Loss, Simulator, Rigid catenary, Arc, Excitation frequency

\section{Introduction}

Various catenary systems have been commercialized because of the development of electric railway technology. The rigid catenary system method was generalized in underground areas. As compared to a catenary, a rigid conductor has advantages such as reduction of maintenance work in underground areas, reduction of operating costs and construction costs due to reduction of the tunnel area, and increased safety, such as the prevention of disconnection accidents. Therefore, the use of rigid conductor systems has expanded [1-2]. Analyzing contact loss is highly important for improving the quality of current collection. The contact force of a catenary that is maintained at the same height changes with an increase in the railway vehicle running speed, and physical contact loss, which is a separation between catenary and pantograph, occurs. This is one of the major issues involved in high-speed electric railways. Because the contact loss occurs when supplying power from the catenary to the pantograph, an arc discharge occurs at the contact surface. This arc causes electrical problems for the railway vehicles and their internal circuits, as well as physical problems such as wear, breakage, and shortened life of the pantograph and overhead wire. The contact loss occurs more because of its low flexibility when the railway vehicle speeds up. Analyzing the interaction between a pantograph and a rigid catenary system is important to mitigate this phenomenon, and a simulator is necessary to analyze its characteristics for speed-up of railway vehicles in rigid catenary system $[3,4]$.

In this study, a contact loss simulator for a rigid catenary

$\dagger$ Corresponding Author: Dept. of Transportation System Engineering, Korea National University of Transportation, Korea.

(goldmoon@ut.ac.kr)

* Dept. of Transportation System Engineering, Korea National University of Transportation, Korea. (bossjng@ut.ac.kr)

Received: November 19, 2016; Accepted: January 16, 2017 system was designed and developed and used to analyze the effect of the power source according to the conditions of the rigid catenary system and pantograph. In the contact loss simulator for the rigid catenary system, R-bar applied to a high-speed train was among the real rigid catenary systems that are used. The excitation frequency generated by the movement of the railway vehicle was simulated. The characteristics according to the frequency and amplitude of the excitation frequency and the presence or absence of pantograph movement were analyzed. This work is considered to be helpful in analyzing the characteristics of contact loss in the interface between a real rigid catenary and rail vehicle.

\section{Characteristics of a Rigid Catenary System}

Fig. 1 shows the sectional view of a rigid catenary system. The rigid catenary system method supports the rigid body by using the free bracket in a narrow place, such as a tunnel or underground area. Because devices such as the tension device and curve push device required in the

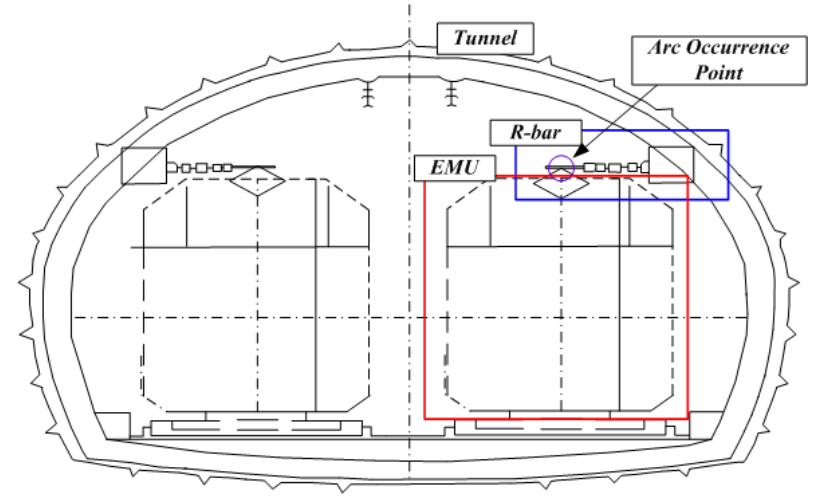

Fig. 1. Cross-sectional diagram of a rigid catenary system 


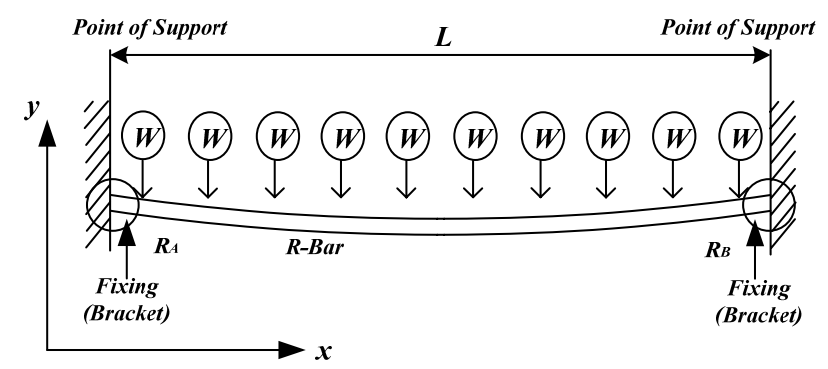

Fig. 2. Deflection phenomenon of a rigid catenary system

catenary suspension method are not required, the device of the rigid catenary system method is simple. In addition, because catenary tension does not exist, there is no risk of broken wires, and because the electrical capacity of the overhead rigid catenary conductor is large, it is possible to supply large-capacity power. However, because the rigid catenary system method compared to the catenary suspension method is less flexible, catenary wear frequently occurs. Because conformability between the pantograph and the overhead catenary is poor, contact loss and arcs frequently occur; sometimes damaging electronic devices in multiple electric units $[5,6]$.

Physical and electrical aspects should be approached in order to analyze the characteristics of the rigid catenary system. Fig. 2 shows the deflection phenomenon of a rigid catenary system. In the middle part of a rigid catenary, the frequency of arc occurrence is high because of the deflection phenomenon. In this study, the deflection of the rigid catenary was calculated from a physical perspective.

Because deflection of a rigid body is generated by gravity in real systems, the $-y$ term is substituted to a new independent variable, $x$ :

$$
\frac{d^{2} v}{d x^{2}}=-\frac{M}{E I}
$$

where $v$ is the deflection of the rigid catenary, $x$ is an arbitrary length, $M$ is the moment, and $E I$ is the flexural stiffness.

On one hand, with the shear force $V$ and moment $M$, the following differential equation is satisfied when the rigid catenary receives any of the distributed load:

$$
\frac{d M}{d x}-V=0
$$

where $V$ is the shear force.

The geometrical moment of inertia of the rigid catenary system expressed as a function of $x$ because the value changes depending on the position. The following equations are derived by substituting (1) into (2):

$$
E I_{x} \frac{d^{2} v}{d x^{2}}=-M
$$

First, it is possible to determine the reaction force of the fixing in Fig. 2. The left and right reaction forces are the same, and the value is the same as (4) because of the uniformly distributed load.

$$
R_{A}=R_{\mathbf{B}}=\frac{w L}{2}
$$

where $R_{A}$ and $R_{\mathbf{B}}$ are the reaction forces, $L$ is the length of the rigid catenary, and $w$ is weight per unit.

The horizontal axis is $x$, and the vertical axis is $y$, as shown in Fig. 2, in order to determine the internal shearing force of the catenary and the bending moment:

$$
\begin{gathered}
\sum F_{y}=0 \\
R_{A}-w x-V=0 \\
V=\frac{w L}{2}-w x
\end{gathered}
$$

where $F_{y}$ is the force applied to an arbitrary point.

Eq. (8) can be derived by substituting (2) into (7), considering the equilibrium in any position $x$ :

$$
M=\frac{w L x}{2}-\frac{w x^{2}}{2}
$$

Eq. (9) can be derived by substituting (1) into (8):

$$
E I \frac{d^{2} v}{d x^{2}}=-M=\frac{w x^{2}}{2}-\frac{w L x}{2}
$$

Eq. (10) can be derived by the double-integration of (9) for calculating the deflection of the rigid catenary:

$$
E I v=\frac{w x^{4}}{24}-\frac{w L x^{3}}{12}+C_{1} x+C_{2}
$$

The integral constant can be derived from the boundary condition. The boundary condition is the same as $v=0$ for $x=0$ and $x=L$, and (11) and (12) can be derived by substituting the boundary condition into (10):

$$
\begin{gathered}
C_{2}=0 \\
\frac{w L^{4}}{24}-\frac{w L^{4}}{12}+C_{1} L=0
\end{gathered}
$$

Therefore, $C_{1}$ is the same as

$$
\therefore C_{1}=\frac{w L^{3}}{24}
$$

The equation of the deflection curve determined by substituting this value is the same as 


$$
v=\frac{w}{24 E I}\left(x^{4}-2 L x^{3}+L^{3} x\right)
$$

The deflection of the rigid catenary occurs as shown in (14), and deformation occurs as a result. Because the copper shoe of a railway vehicle slides, the catenary incurs deflection or deformation, causing excitation and contact loss of the catenary. The maximum deflection occurs in the middle of the rigid catenary, and its value is the same as

$$
\delta=v\left(\frac{L}{2}\right)=\frac{5 w L^{4}}{384 E I}
$$

where $\delta$ is the maximum deflection.

As shown in (15), the maximum deflection becomes smaller as the bending stiffness of the rigid catenary increases because of its weight. It can also be seen that deflection increases proportionally according to the fourth power of the length. The arc is generated in some of the impact between the pantograph of railway vehicle and catenary. If the speed of the railway vehicle is high, this phenomenon occurs frequently.

\section{Definition of Contact Loss and Characteristics of Arc}

Contact loss can result in abnormal wear and damage by causing arcs because of electrically incomplete contact. Therefore, the contact loss occurring in motion is inevitably one of the key factors to determine the speed of a train. The rate of loss of contact is defined as the "percentage of contact loss." Percentage of contact loss is (the sum of contact loss time when driving time of specific section) / (driving time of specific section) $* 100 \%$.

\subsection{UIC 799 OR and IEC 62486}

Contact loss is defined in UIC 799 OR as "the arc over contact loss time $10 \mathrm{~ms}$ per $100 \mathrm{~m}$ distance." This excludes a maximum of $25 \mathrm{~ms}$ and above [7]. And contact loss is defined in IEC 62486 as an arc of $5 \mathrm{~ms}$ and over among current time when more than $30 \%$ of the rated current is flowing. In addition, contact loss is less than $0.1 \%$ in sections below $250 \mathrm{~km} / \mathrm{h}$ and less than $0.2 \%$ in sections above $250 \mathrm{~km} / \mathrm{h}$, and should not exceed the maximum of $0.5 \%[8]$.

\subsection{Electrical characteristics of ares}

The arc phenomenon refers to exceeding the dielectric strength of the air between two electrodes; the insulation is destroyed and electrical discharge current flows. High temperature and light are emitted by the electrical discharge between the two electrodes. Joule heat generated at this time is represented by the formula $I^{2} R t$. Discharge energy $e$ generated by the arc current is expressed as the integral of the product of the discharge current $I$ and arc voltage $V$. When considering the arc start time of a onetime occurrence during the half-period $t_{1}$ and the end time of the arc $t_{2}$, the discharge energy $e$ of the arc current is the same as [9]:

$$
e=\int_{t_{1}}^{t_{2}} V I d t
$$

Arcs generated in electric railway feeding lines can affect the feeding system, reducing the lifetime for internal devices of the vehicle, and generate harmonics and EMI, which in turn degrade the stability of the facility. A harmonic incoming to the feeding system by an arc leads to load losses, requiring proper review of the capacity of the existing facility. In addition, because of inductive disturbances generated by installation methods and harmonic current, a correction to the allowable current of the wire is necessary. In addition, because noise may occur in the railway signaling system, the problem is serious; in addition, the electric railway vehicle is supplied with electric power using the pantograph and risks malfunction of the electromagnetic wave because it is used in a variety of power conversion systems. Electromagnetic waves in an electric railway are generated by the switching surge of the power conversion system, the corona, and the arc. The main cause of the generated dual-electromagnetic interference is arcs by contact loss between the catenary and the pantograph.

\section{Design of Contact Loss Simulator for Rigid Catenary System}

First, theoretical analysis of the arc is necessary in order to analyze the arc using the contact loss simulator for the rigid catenary system. In this study, the contact loss simulator for a rigid catenary system to generate contact loss was designed and its operational characteristics were analyzed.

The pantograph slides on the catenary in an electric railway vehicle, and vibration occurs according to the excitation frequency in the catenary. The simulator in this study is a prototype model. Because generating the vibration is difficult according to the running of the railway vehicle, in order to generate the contact loss, a motor was installed at the left end of the rigid catenary, and a system was installed to generate vibrations through vertical movement according to the frequency. Vibration is designed to be $0 \sim 10 \mathrm{~Hz}$ [6] in the frequency effect characteristics of the current collection of the pantograph, and the experiment was performed by varying the conditions. For the study of the interface of the rigid catenary system, in order to simulate the contact loss in the rigid catenary system, the 
Table 1. Parameters of contact loss simulator for the rigid catenary system

\begin{tabular}{|c|c|c|c|}
\hline \multicolumn{2}{|c|}{ Content } & $\begin{array}{l}\text { High-speed } \\
\text { railway }\end{array}$ & $\begin{array}{c}\text { Contact loss } \\
\text { simulator }\end{array}$ \\
\hline \multicolumn{2}{|c|}{ Input voltage } & $25,000 \mathrm{~V}$ & $220 \mathrm{~V}$ \\
\hline \multicolumn{2}{|c|}{$\begin{array}{c}\text { MCB } \\
\text { breaking current } \\
\end{array}$} & $2,000 \mathrm{~A}$ & $20 \mathrm{~A}$ \\
\hline \multicolumn{2}{|c|}{$\begin{array}{c}\text { Maximum capacity of } \\
\text { power conversion system }\end{array}$} & $2,600 \mathrm{~kW}$ & $2.0 \mathrm{~kW}$ \\
\hline \multirow{2}{*}{ R-bar } & Material & \multicolumn{2}{|c|}{ Aluminum Alloy 6063} \\
\hline & Radios & \multicolumn{2}{|c|}{$0.0265 \mathrm{~m}$} \\
\hline
\end{tabular}

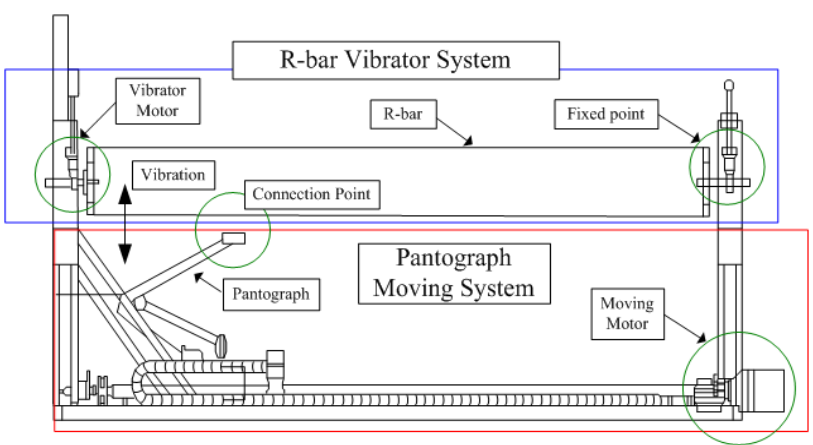

Fig. 3. Contact loss simulator for the rigid catenary system

parameters shown in Table 1 were applied.

Fig. 3 shows a block diagram of the simulator for contact loss analysis. The simulator is divided into a vibrator system for the rigid catenary and a movement system for the pantograph. R-bar was installed at the top of the simulator, and the pantograph was installed at the bottom of the simulator in order to simulate contact loss in the rigid catenary system while running the railway vehicle.

In addition, as the pantograph slides along the rigid conductor, the system is capable of controlling the moving push-up force of the pantograph. The transient characteristics of the contact loss simulation were close to the actual characteristics and were applied to enable the vibration of the excitation frequency.

Fig. 3 illustrates the contact loss for the rigid catenary system. A rigid catenary was used for the R-bar applied to high-speed rail. The fixed point is at the right end of the top, and a motor to enable to vibrations in the desired frequency band and amplitude was installed at the left end of the top in order to simulate the R-bar vibration. In addition, a motor to enable pantograph motion to the left and right side was installed at the bottom of the pantograph motion system. The air pressure of the pantograph was controlled through the compressor control, which was designed to control the push-up force of the pantograph.

Fig. 4 shows that arcing occurs when spacing between the R-bar and the pantograph is instantaneously generated.

In this study, the contact loss was generated through the vertical vibration of the rigid catenary system at a specific frequency. It was designed to simulate the phenomenon of contact loss by changing the frequency of the vertical
Table 2. Specifications regarding changes of frequency and amplitude in the simulation

\begin{tabular}{c|c}
\hline Content & Range of change \\
\hline frequency & $2 \sim 8 \mathrm{~Hz}$ \\
\hline amplitude & $5 \sim 15 \mathrm{~mm}$ \\
\hline Speed & $600 \sim 1000 \mathrm{~m} / \mathrm{h}$ \\
\hline
\end{tabular}

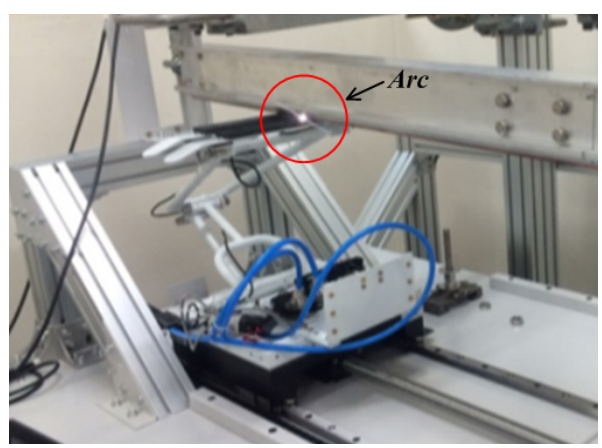

Fig. 4. Arcing between R-bar and pantograph

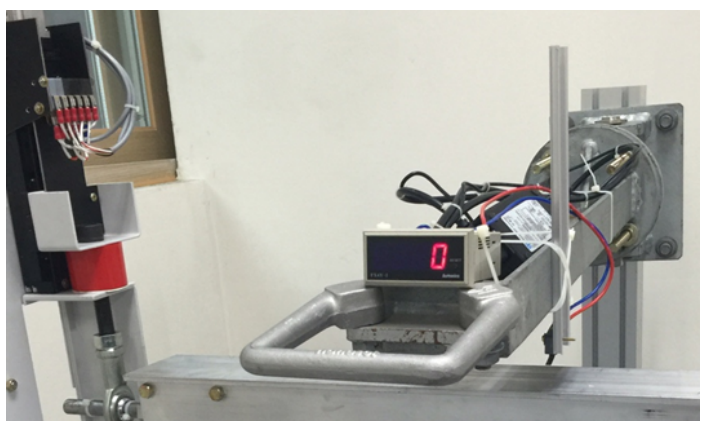

Fig. 5. Counter for calculation of R-bar frequency

vibration.

Table 2 shows specifications regarding changes in frequency and amplitude in the simulation.

A counter was installed on top of the R-bar as shown in Fig. 5 to measure the frequency of the R-bar. Counts per unit time was obtained by using the counter and the R-bar frequency was calculated. Frequency was measured over 1 $\min$. The frequency $f$ can be derived from

$$
f=\frac{\text { Count }}{T}
$$

where Count is the value displayed on the counter.

Fig. 6 shows the software process of the contact loss simulator. When the controller of the contact loss simulator for the rigid catenary system is operated, the initial state is checked through operational checks and the pantograph speed; the R-bar up-and-down frequency and stroke are controlled according to the input signal. Initial control confirms the initial position in the respective situation and starts the operation after moving to the origin. If the 


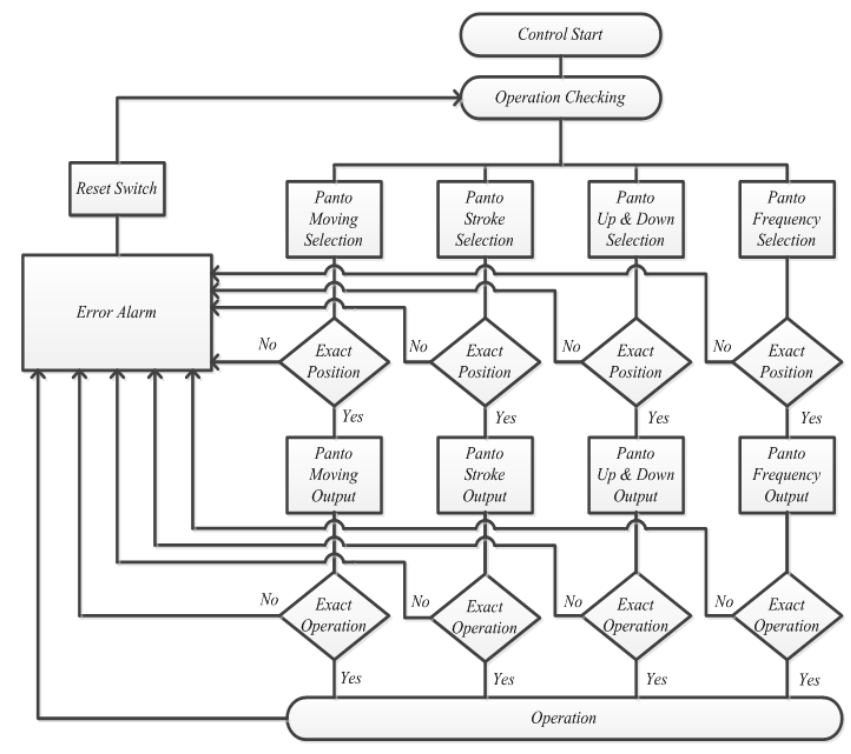

Fig. 6. Software process of the contact loss simulator

pantograph is not in the correct position or it does not operate correctly in the respective situation, the operation was designed to stop with the error alarm. In this situation, it was designed to operate through a reset switch.

\section{Analysis of the Contact Loss Phenomenon using the Rigid Catenary System}

In this study, contact loss was analyzed in accordance with the movement of the pantograph. Because electric railway vehicles use induction motors or synchronous motors, an inductor is essentially applied. Therefore, experiments were performed in Resistance - Inductor serial (R-L) load conditions. And the contact loss was found in zero point of voltage or current. This is because there is no place for current to flow.

Fig. 7 shows the voltage and current waveforms in the stationary condition with $2 \mathrm{~Hz}$ frequency, $5 \mathrm{~mm}$ amplitude, and R-L load. Fig. 8 shows the zoomed waveform of a contact loss point in Fig. 7. Constant contact losses occur in the stationary condition.

Fig. 9 shows the voltage and current waveforms in the 0.6-km/h moving condition, with $2 \mathrm{~Hz}$ frequency, $5 \mathrm{~mm}$ amplitude, and R-L load. Fig. 10 shows the zoomed waveform of the contact loss point in Fig. 9.

The voltage and current waveforms in the moving condition had no contact loss during the $6.4 \mathrm{~s}$ based fixed point (total time of the waveform is $12.8 \mathrm{~s}$ ), but contact loss occurred after this time. The zoomed waveform analysis of Fig. 8 and Fig. 10 is as follows: It can be seen that the waveform distortion of the moving condition is more severe than that of the stationary condition, because the contact point in the moving condition is more unstable than that of the stationary condition.

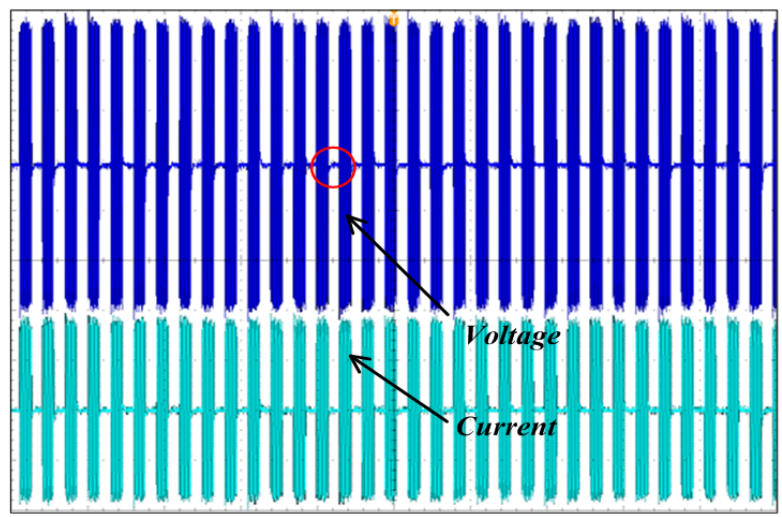

(X axis: 2 s; Y axis: $100 \mathrm{~V} /$ div., $10 \mathrm{~A} /$ div.)

Fig. 7. Voltage and current waveforms in the stationary condition

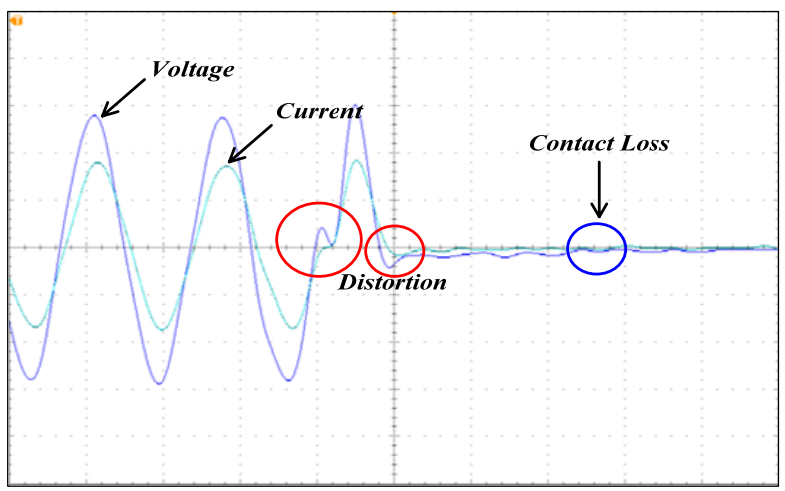

(X axis: $10 \mathrm{~ms}$; Y axis: $100 \mathrm{~V} /$ div., $10 \mathrm{~A} /$ div.)

Fig. 8. Zoomed waveform of Fig. 7 in the stationary condition

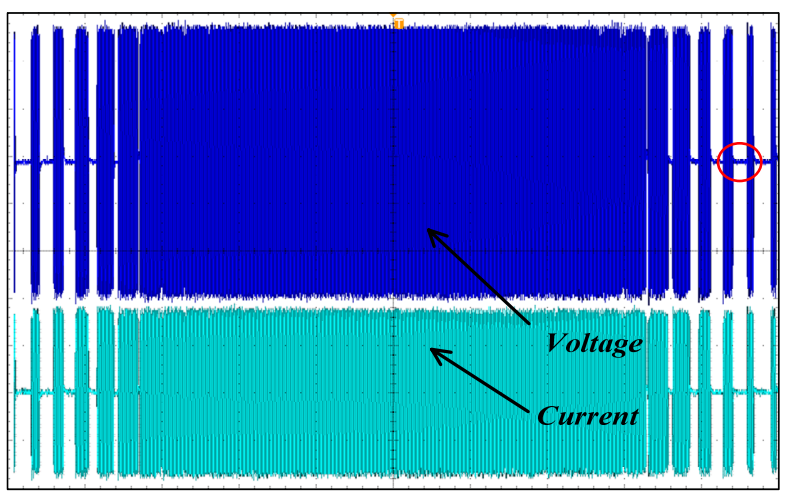

(X axis: $2 \mathrm{~s}$; $\mathrm{Y}$ axis: $100 \mathrm{~V} /$ div., $10 \mathrm{~A} /$ div.)

Fig. 9. Voltage and current waveforms in the moving condition

\subsection{Analysis of the contact loss phenomenon according to $\mathbf{R}$-bar amplitude}

The waveform was analyzed in the condition of R-L load, constant frequency, and the pantograph in the moving state for the contact loss phenomenon analysis according to 


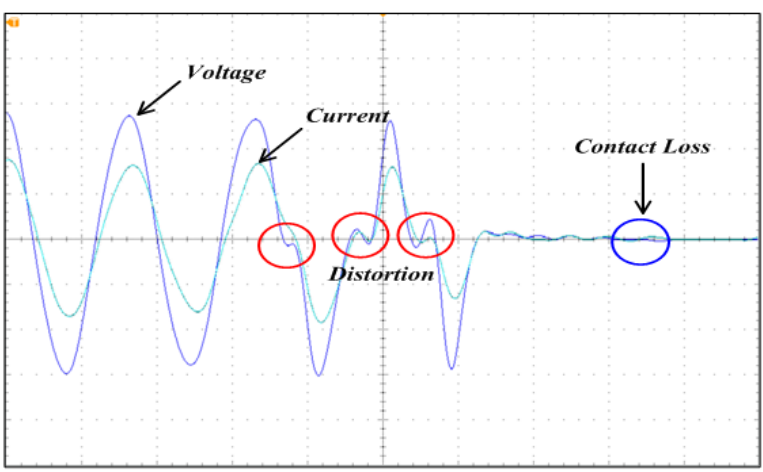

( $\mathrm{X}$ axis: $10 \mathrm{~ms}$; $\mathrm{Y}$ axis: $100 \mathrm{~V} /$ div., $10 \mathrm{~A} /$ div.)

Fig. 10. Voltage and current zoomed waveform in the moving condition

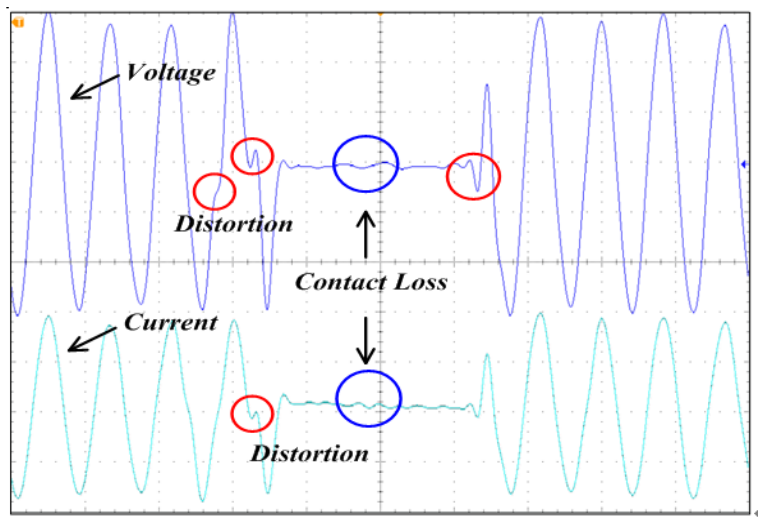

(a) 5-mm amplitude

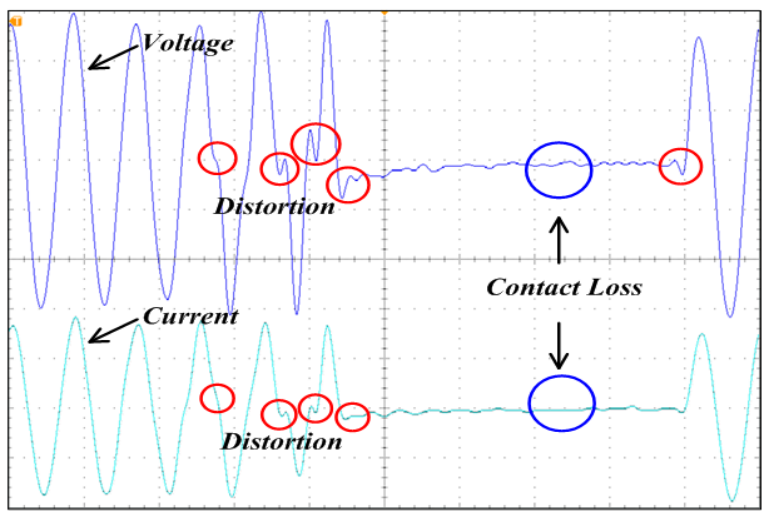

(b) 15-mm amplitude

(X axis: $20 \mathrm{~ms}$; $\mathrm{Y}$ axis: $100 \mathrm{~V} /$ div., $10 \mathrm{~A} /$ div.)

Fig. 11. Voltage and current waveforms for different amplitudes

amplitude variation. Fig. 11 shows the voltage and current waveforms according to amplitude in the $0.6-\mathrm{km} / \mathrm{h}$ moving condition with $2 \mathrm{~Hz}$ frequency and R-L load.

It can be confirmed that the waveform distortion for the $15-\mathrm{mm}$ amplitude is more severe than that of $10-\mathrm{mm}$ amplitude, as shown in Fig. 11. This is because the force with which the R-bar hits the pantograph in the $15-\mathrm{mm}$

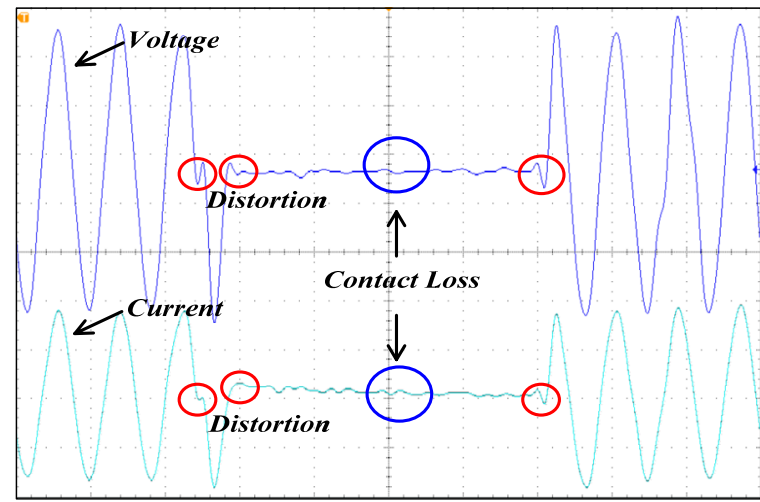

(a) $2-\mathrm{Hz}$ frequency

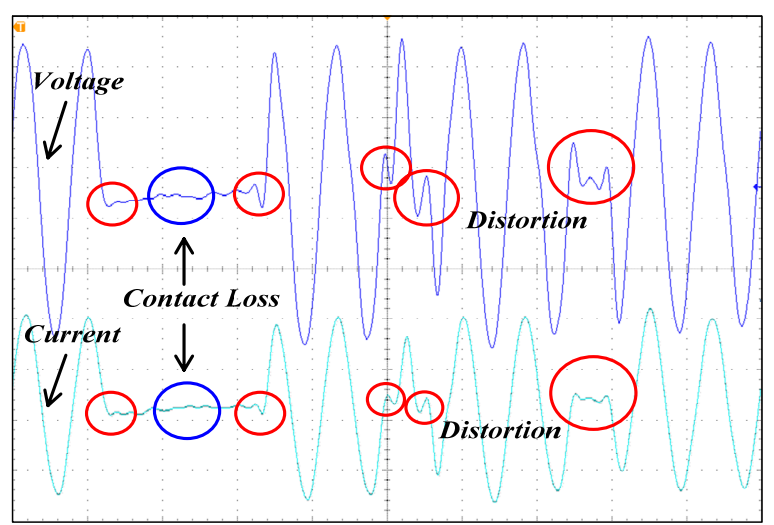

(b) 5-Hz frequency

(X axis: $20 \mathrm{~ms}$; $\mathrm{Y}$ axis: $100 \mathrm{~V} /$ div., $10 \mathrm{~A} /$ div.)

Fig. 12. Voltage and current waveform for different frequencies

condition is stronger than that of $10 \mathrm{~mm}$.

\subsection{Analysis of the contact loss phenomenon according to R-bar excitation frequency}

The waveform was analyzed in the condition of R-L load, constant amplitude, and pantograph moving state for the contact loss phenomenon analysis according to frequency variation. Fig. 12 shows the voltage and current waveforms according to frequency in the $0.6-\mathrm{km} / \mathrm{h}$ moving condition, $5 \mathrm{~mm}$ amplitude, and R-L load.

Comparing Figs. 12(a) and 12(b) confirms that the waveform distortion at $5 \mathrm{~Hz}$ is more severe than that of 2 Hz. The higher the frequency, the greater distortion. In addition, the waveform distortion was worse at $5 \mathrm{~Hz}$ as compared to that at $2 \mathrm{~Hz}$ after contact loss, because the Rbar is impacted again in the state that the previous frequency was not completely removed.

\subsection{Analysis of experimental results}

In this study, the experiment was performed using an R-L load to simulate the railway vehicle load. The characteristics were analyzed according to the change of 
Table 3. Average number of distortions of the voltage waveform for each condition

\begin{tabular}{|c|c|c|c|}
\hline Frequency $\quad$ Amplitude & $5 \mathrm{~mm}$ & $10 \mathrm{~mm}$ & $15 \mathrm{~mm}$ \\
\hline $2 \mathrm{~Hz}$ & 2.8 & 3 & 4.2 \\
\hline $5 \mathrm{~Hz}$ & 4.5 & 4.8 & 4.6 \\
\hline $8 \mathrm{~Hz}$ & 5.3 & 5.3 & 5.4 \\
\hline
\end{tabular}

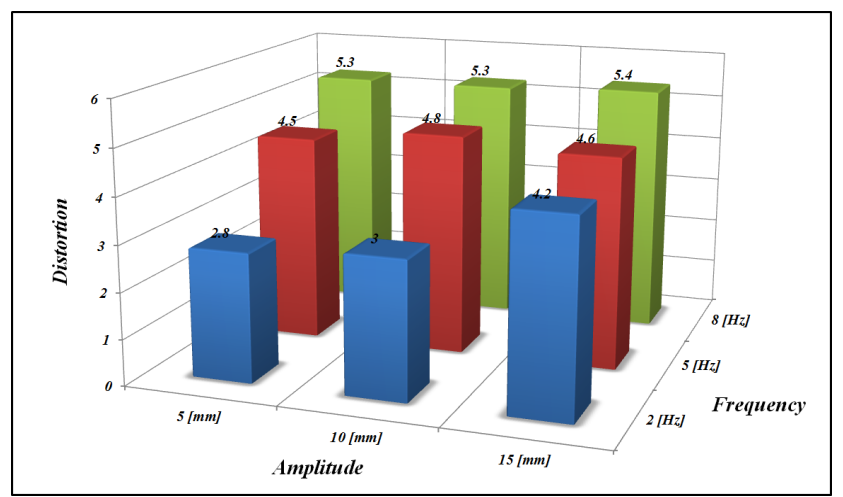

Fig. 13. Distortion of the voltage waveform for each condition

frequency and amplitude in the moving condition. Because the voltage and current flow is irregular, THD cannot be measured. Table 3 shows the average value of the number of waveform distortions by performing the experiment 10 times in each condition.

From Table 2, the waveform distortions increased with increasing amplitude at $2 \mathrm{~Hz}$, but there was no large change at $5 \mathrm{~Hz}$ or $8 \mathrm{~Hz}$. The waveform distortions generally increased with increasing frequency. It can be seen that the distortion reacts more sensitively at a frequency change from 2 to $8 \mathrm{~Hz}$ than an amplitude change from 5 to $15 \mathrm{~mm}$.

\section{Conclusion}

In this study, the characteristics were analyzed through the design and manufacture of a rigid-bar contact loss simulator used in a real railway system. The waveform was analyzed according to changes in amplitude and frequency. The results of the study are as follows:

(1) It was confirmed that the waveform distortion in the moving condition is more severe than that of the stationary condition. This is because the contact point in the moving condition is more unstable than that of the stationary condition.

(2) It was confirmed that the waveform distortion of 15 $\mathrm{mm}$ amplitude is more severe than that of $10 \mathrm{~mm}$ amplitude. This is because the force with which the R-bar hits the pantograph in the $15-\mathrm{mm}$ amplitude condition is stronger than that of the $10-\mathrm{mm}$ amplitude condition.

(3) The higher the frequency, the more distortion; the distortion of the waveform was worse at $5 \mathrm{~Hz}$ compared to
$2 \mathrm{~Hz}$ when contact loss started. This is because the R-bar is impacted again in the state where the previous frequency was not completely removed.

(4) It can be seen that the distortion reacts more sensitively at a frequency change from 2 to $8 \mathrm{~Hz}$ than an amplitude change of 5 to $15 \mathrm{~mm}$, from the results of multiple experiments.

This work will be useful for analyzing the characteristics of the line between the R-bar and railway vehicle interface.

\section{Acknowledgements}

This research was supported by the IRCPRC (Inventive Railway Components and Parts Research Center).

\section{References}

[1] Y. Park et al, "Reliability Analysis of Arcing Measurement System Between Pantograph and Contact Wire," Transactions of the Korean Institute of Electrical Engineers, vol. 61, no. 8, pp. 1216-1220, 2012.

[2] N.-G. Jung et al, "A Study on Characteristic of Power Conversion System in Electric Railway Vehicle According to Contact Loss in Feeding System Considering Characteristic of Rigid Bar," Transactions of the Korean Institute of Electrical Engineers, vol. 65, no. 3, pp. 520-525, 2016.

[3] J. M. Kim et al, "Transient Characteristic Study on Contact Loss of High Speed Electric Railway Using a Power Line Disturbance Simulator," Transactions of the Korean Institute of Electrical Engineers, vol. 58, no. 4, pp. 427-431, 2009.

[4] J. M. Kim et al, "Development of simulator by induced contact loss phenomenon for high-speed train operation," in Conference on Korean Society for Railway, 2009.

[5] Y. S. Han, "A Study on Arc Characteristics between pantograph and contact wire of Electric Railroad," Master's thesis, 2010.

[6] S. J. Bae et al, "A Study on Characteristics of Overhead Rigid Conductor System for Developing the High-speed System up to $250 \mathrm{~km} / \mathrm{h}$," Transactions of the Korean Institute of Electrical Engineers, vol. 64, no. 3, pp. 492-497, 2015.

[7] UIC 799 OR, "Characteristics of a.c. overhead contact systems for high speed lines worked at speeds of over $200 \mathrm{~km} / \mathrm{h}, " 2002$.

[8] IEC 62486, "Current collection systems - Technical criteria for the interaction between pantograph and overhead line (to achieve free access), 2010.

[9] Hwi Ju Kim, "A Study on the Contact Loss Arc Property of Railway Power Feeding System," Master's thesis, 2016. 


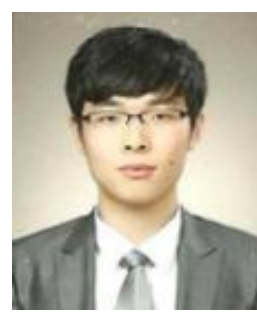

No-Geon Jung received the B.S. degree in rolling stocks electric engineering from the Korea National Railroad College, Korea, in 2012, and then received M.S. degree from Korea National University of Transportation in 2015, Since 2015, he has been with Korea National University of Transportation, where he is currently a Ph.D. course. His research interest is power electronics of railway.

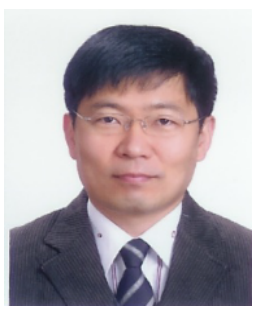

Jae-Moon Kim received the B.S degree in 1994 and the Ph.D. degree in 2000 from Sungkyunkwan University. From 2004 to 2011, he was with the department of rolling stock electrical engineering, Korea National Railroad College. Since 2012, he has been with the department of transportation engineering in the transportation graduated school, KNUT. His research interests are power conversion in industry fields, traction and regeneration control in electric railway. 